\title{
III. Ein Fall von Morbus Basedowii, geheilt durch eine Operation in der Nase. ${ }^{1}$ )

\author{
Von Dr. A. Musehold in Berlin.
}

Die höchst bewerkenswerthe Thatsache, dass nach Beseitigung von Nasenleiden Heilung der Basedow'schen Krankheit eintrat, ist bisher selten beobachtet. Der erste Fall wurde im Jahre 1885 von Hopmann auf der 58. Versammlung deutscher Naturforscher und Aerzte zu Strassburg mitgetheilt und später nochmals in extenso in der Berliner klin. Wochenschrift 1888 No. 42 veröffentlicht. Er betraf eine 40jährige Frau mit incompletem Morbus Basedowii es fehlte die Struma - , welche ausserdem an Rhinopharyngitis sicca litt und kleine Polypen an der rechten mittleren Muschel zeigte. Nachdem bereits die Beseitigung der sehr reichlichen Borken durch die Gottstein'sche Methode erhebliche Besserung des Allgemeinbefindens zur Folge hatte, trat ein schneller Rückgang der Krankheitserscheinungen in unmittelbarem Anschluss an die Entfernung der Schleimpolypen ein. Ein leichter Rückfall wurde durch abermalige energische Behandlung der Rhinopharyngitis beseitigt. In dem zweiten Fall, von Hack (Deutsche medicin. Wochenschr. 1886 No. 25), handelte es sich um eine 17jährige junge Dame mit vollzähliger Symptomentrias. Die grössten Beschwerden verursachte durch Hyperplasie der unteren Muscheln bedingte Nasenstenose. Bereits am folgenden Tage nach der galvanokaustischen Operation in der rechten Nase war der Exophthalmus rechts nahezu verschwunden; fast ebenso schnell bildete sich die Protrusio bulbi links nach dem entsprechenden Eingriff in der linken Nasenhälfte zurück, während im übrigen zur völligen Heilung mehrere Monate erforderlich waren. Drittens stellte B. Fraenkel in der Berliner medicinischen Gesellschaft (Berliner klin. Wochenschr. 1888 No. 6) einen jungen Mann vor, bei welchem von den Cardinalsymptomen der Exophthalmus fehlte. Im Verlaufe der Behandlung mit dem constanten Strom klagte der Kranke über doppelseitige Nasenstenose. Unmittelbar nach jedem galvanokaustischen Eingriff trat eine rapide Abschwellung des Kropfes der entsprechenden Seite ein. Gleich nach der ersten Operation wurde der Puls langsamer, und etwa 4 Tage nach der zweiten normal. Ein Einfluss des Elektrisirens ist hier auszuschliessen, da in der dreiwöchentlichen Pause zwischen den beiden chirurgischen Eingriffen die Kropfhälfte der noch nicht operirten Seite trotz Fortsetzung der elektrischen Behandlung unverändert blieb. Von grossem Interesse dürften weiterhin noch zwei von Stoker beobachtete Fälle sein, bei denen beträchtliche Struma nach mehrfachen galvanokaustischen Aetzungen in der Nase sich zurückbildete (Internat. Centralblatt für Laryngologie V. 579). Endlich liegt eine hervorragende Beobachtung von Semar vor, wo umgekehrt in 1-3 Tagen nach einer galvanokaustischen Operation in der Nase zunächst einseitiger Exophthalmus mit dem Graefeschen Symptom auftrat, und bald darauf auch noch die anderen Erscheinungen der Basedow'schen Krankheit, ausser Struma. Nach weiteren Operationen zeigte sich Rückbildung der Symptome (Internat. Centralbl. für Laryngologie VI. 238).

1) Nach einem Vortrage, gehalten in der Berliner Laryngologischen Gesellschaft. 
Diesen Beobachtungeu kann ich eineu neuen Fall hinzufügen, dessen Krankengeschichte ich hier folgen lasse:

Frau Vocke, verheirathete Plätterin, ist 45 Jahre alt und war bis zu ihrem 40. Lebensjahre vollkommen gesund. In diesem Alter begann sie mit dem Eintritt der Menopause über Kopfschmerzen zu klagen, welche im ganzen unregelmässig auftraten, jedoch in den Tagen der früheren Periode sich zu unerträglicher Heftigkeit steigerten. Ungefähr eiı Jahr später nahm die Kranke an Körperfülle zu, doch veränderte sich damit die frühere frische Gesichtsfarbe in eine auffallend blasse, so dass die Kranke, nach ihren eigenen Worten, wie gedunsen aussah. Bald darauf, also etwa $1^{1} / 2$ Jahre nach der letzten Menstruation stellte sich öfter Herzklopfen mit stechenden Schmerzen in der Herzgegend ein und wurde namentlich nach Genuss von Caffee oder Bier sehr heftig. Unter allmählicher Zunabme desselbeu begann die Frau abzumagern, sie wurde ausserordentlich leicht erregbar und zeigte verdriessliche Stimmung. Sie fühlte sich zunehmend schwächer und bemerkte, dass ihr die Arme und Hände, besonders die rechte Hand, zitterten, "selbst wenn sie dieselben ruhig auf den Tisch legte." Kurze Zeit nach dem Auftreten des Herzklopfens machte sich eine allmählich wachsende Schwellung vorn am Halse bemerkbar. Trotz aller Beschwerden hat die Kranke ihre anstrengende Arbeit als Plätterin noch verrichten können bis etwa zum Herbst vergangenen Jahres. Seit dieser Zeit haben die Kopfschmerzen, das Herzklopfen, die leichte Erregbarkeit und Mattigkeit so zugenommen, dass die Kranke, besonders in den letzten Monaten, oft gezwungen war, ihre Arbeit zu unterbrechen und durch Ruhe Besserung ihres Zustandes zu erzielen. Das Herzklopfen war zweimal, im December 1890 und Juli dieses Jahres, und zwar Nachts mit so heftiger Athemnoth aufgetreten, dass die Kranke vor Angst aus dem Bette sprang und am geöffneten Fenster Erleichterung suchte. Als neue Beschwerden zeigten sich im Winter 1890/91 zunächst vorübergehende Schwellungen beider Füsse bis zu den Knöcheln, ferner schliefen ihr Nachts - die Kranke pflegt auf der rechten Seite zu liegen - auffallend oft der rechte Arm und das rechte Bein ein; das letztere in so hohem Grade, dass das unangenehme Gefühl in Schmerzen ausartete. Ganz besonders lästig aber wurde ihr eine in diesem Frühjahr zuerst aufgetretene Neigung zu profusen Schweissen, sowohl bei Tage als auch - und zwar in hervorragendem Maasse - in der Nacht. Der Kropf hatte sich inzwischen allmählich so vergrössert, dass die vor zwei Jahren gemessene Halsweite von $38 \mathrm{~cm}$ auf $40 \mathrm{~cm}$ gestiegen war. Zu allem kamen seit Juni noch Beschwerden über das rechte Auge: lästiges Thränentrüufeln, ferner, namentlich bei künstlichem Licht, Schmerzen in dem Auge und Flimmern, so dass die Kranke an der Abendbeschäftigung verhindert wurde. Diese letzten Klagen veranlassten sie, die Poliklinik für Augenkranke des Herrn C. du Bois-Reymond aufzusuchen. Da in denselben Räumen sich auch mein Ambulatorium befindet, so pflegt Herr du Bois-Reymond Kranke mit bestimmten Augenleiden auch mir zur Untersuchung der Nase und eventuell weiteren Behandlung zu überweisen. Das geschah auch in dem vorliegenden Falle, und ich nehme an dieser Stelle gern Gelegenheit, dafür meinen verbindlichsten Dank besonders auszusprechen.

Als ich die Kranke am 3. August zum ersten male sah, klagte sie zunächst über das rechte Auge, fügte aber gleich hinzu, dass ihre Hauptleiden täglich auftretendes heftiges Herzklopfen und quälende Kopfschmerzen seien. Auf Befragen gab sie an, dass der Kopfschmerz, unabhängig von dem Herzklopfen, an der Nasenwurzel beginne und sich über die Stirn bis zu den Schläfen verbreite. Die ziemlich grosse Patientin war mager, auffallend blass, ihre Augen hatten einen glasigen Glanz, die Conjunctivae bulbi einen bläulichen Schimmer. Die Conjunctivae der Augenlider zeigten leichte Hyperämie. Die Pupillen waren gleich weit, reagirten auf Lichtreiz gleichmässig normal. Eine Protrusion der Augäpfel konnte ich nicht bemerken, obwoh! das rechte Auge ein wenig mehr vorzustehen schien, als das linke. Druck auf die Bulbi war nicht schmerzhaft, das Gräfe'sche Symptom fehlte. - Am Halse fiel gleich eine ziemlich starke Struma auf, welche in ihrer rechten Hälfte sichtlich grösser war, als links. Sie war diffus, fühlte sich ziemlich weich an; Gefässgeräusche konnten nicht wahrgenommen werden. Der Puls war beschleunigt, etwa 90-100, sehr unregelmässig und ziemlich klein. Die Untersuchung des Herzens ergab zunächst keine sichtbaren Hebungen in der Herzgegend, besonders nicht an der Stelle des Spitzenstosses, welcher innerhalb der Mamillarlinie im 5. Intercostalraum schwach fühlbar war. Die Herztöne waren rein, ebenso konnten über den Halsgefässen nur zwei schwache reine Töne gehört werden. Die Lungen erwiesen sich als gesund. Bei vorgestreckten Unterarmen und Händen mit gespreizten Fingern zeigt sich Andeutung von Tremor, rechts mehr, als links.

Die Rhinoscopia anterior ergab: sehr blasse Schleimhaut; die rechte untere Muschel war in ihrer hinteren Hälfte geschwellt, so dass die Wegsamkeit des unteren Nasenganges ziemlich beeinträchtigt wurde. Nach Cocainisirung zeigte sich eine mässige Hyperplasie des hinteren Endes der Muschel. Dasselbe ergab die Untersuchung vom Rachen aus. Subjective Klagen über die Nase bestanden nicht. Die Kranke gab auf Befragen an, dass sie wohl öfter an Schnupfen leide, im übrigen aber auf die Nase nicht geachtet habe.

Der positive Befund in der Nase und die bestimmte Angabe der Kranken, dass der Kopfschmerz von der Nasenwurzel ausgehe, liessen es möglich erscheinen, dass letzterer mit der Nasenaffection in Zusammenhang stehe. Ebenso liess mich die Thatsache, dass die Augenbeschwerden sich auf die Seite beschränkten, wo auch die erwähnte Hyperplasie der unteren Musche! vorhanden war, auch in dieser Richtung auf guten Erfolg einer galvanokaustischen Operation hoffen; zumal es mir in letzter Zeit in einigen Fällen gelungen war, durch galvanokaustische Beseitigung von Schwellungen, beziehungsweise Cristae, lange dauerndes Thränen in wenigen Tagen zum Schwinden zu bringen. Ich rieth der Kranken mit Rücksicht auf ihr Allgemeinbefinden, sich zu schonen, verordnete ein leichtes Eisenpräparat und entfernte am folgenden Tage, 4. August, mit der Glühschlinge das hyperplastische hintere Ende der rechten unteren Muschel in der Grösse einer mittelgrossen Bohne. - Damit war die Kranke, wie mit einem Schlage, von den Kopf- schmerzen befreit, die Augenbeschwerden waren am nächsten Tage geringer und nach weiteren 3 Tagen geschwunden. Ferner aber trat das Herzklopfen an den 3 folgenden Tagen in geringerem Grade und seltener auf, als früher. Vom 5. Tage an blieb dasselbe ganz fort, und am 7. Tage nach der Operation konnte ich bereits notiren: kein Herzklopfen mehr, auch nach Genuss von Caffee nicht. Ich hatte nämlich, nachdem das Herzklopfen 2 Tage ausgeblieben war, die Kranke aufgefordert, zur Probe wieder Caffee zu trinken.

Nach diesem erstaunlichen Erfolge hoffte ich auch auf eine baldige Verkleinerung der Struma, wie sie bereits in den Fällen von Back, B. Fraenke! und Stoker beobachtet war. Doch glaubte ich dieselbe nicht thatlos abwarten zu dürfen, sondern begann am 7. Behandlungstage, den 11. August, mit der Anwendung des constanten Stroms. Ich stellte denselben, da die Kranke sehr empfindlich war, ganz schwach, 1-2 M. A., so dass ihn die Kranke bei der Unterbrechung eben fühlen konnte. Die Dauer jeder Sitzung betrug 5-7 Minuten, und zwar elektrisirte ich zunächst einen Tag um den andern, später 2 mal in der Woche. Bereits am 22. August, also in der 5. Sitzung, erklärte mir die Kranke mit Bestimmtheit, dass sie eine Abschwellung des Kropfes bemerke, der Kragen ihres Kleides schliesse nicht mehr so fest, wie vor Beginn der Behandlung. Am 27. August musste ich plötzlich verreisen und somit die elektrische Behandlung nach, im ga izen, Sitzungen unterbrechen.

Erst am 19. September, also etwa 4 Wochen später, sah ich die Kranke wieder. Das Aussehen derselben war im allgemeinen noch unverändert, die früheren Beschwerden hatten sich jedoch nicht wieder gezeigt. Die Struma war zu meinem Erstaunen so wesentlich verkleinert, dass die Kranke sich inzwischen ihre Kragen enger machen lassen musste, um sich vor Erkältung zu schützen. Der Halsumfang war von $40 \mathrm{~cm}$ auf $36,5 \mathrm{~cm}$ herabgesunken! Die Herzaction war noch unregelnässig, jedoch von normaler Frequenz. Trotzdem die Kranke über diesen Erfolg sehr glücklich war, so beeinträchtigte doch noch leichte Verdriesslichkeit und Mattigkeit ihre völlige Zufriedenheit. Ich liess nun Liquor Ferri albuminati weiternehmen, das inzwischen von der Kranken ausgesetzt war, musste jedoch bereits nach 7 Tagen vorübercehend davon absehen, weil sich Magenbeschwerden eingestellt hatten. Nach Beseitigung der letzteren war das Allgemeinbefinden erheblich besser geworden, die Kranke jetzt zufriedener, als je. Gleichwoh! veranlasste ich die Patientin - am 29. September -, sich auch von einem Frauenarzt untersuchen zu lassen, zumal eine am 14. Behandlungstage aufgetretene Blutung, welche von der Kranken für die nach 5 Jahren wiedergekehrten Menses gehalten wurde, zur Klärung des Krankheitsbildes aufforderte. Die Kranke wandte sich an Herrn Dr. Pyrkosch, welcher einen Cervixkatarrh constatirte und nach 4 wöchentlicher Behandlung Heilung desselben erzielte.

Das Allgemeinbefinden der Kranken ist jetzt ein vorzügliches. Sie ist vollständig arbeitsfähig, ihr Aussehen hat sich verbessert, sie nimmt auch an Körperfülle zu. Von den früheren Krankheitserscheinungen ist gegenwärtig nur noch eine ganz geringe Vergrösserung des rechten Schilddrüsenlappens übrig, die Unregelmässigkeit der Herzaction inzwischen auch geschwunden.

Dass bei der Kranken wirklich Morbus Basedowii vorgelegen hat, dürfte keinem Zweifel unterliegen. Das Fehlen des deutlichen Exophthalmus, welcher der Glotzangenkrankheit einst den Namen gab, ist, wie auch der Ausfall je eines der beiden anderen Cardinalsymptome, vielfach beobachtet, ohne dass dadurch die Diagnose fraglich geworden wäre. In dem vorliegenden Falle stützte sich die Diagnose auf die objectiv wahrnehmbaren Symptome: das blasse Aussehen, die glasigen Augen, das Thränen des rechten Auges, die Struma, die beschleunigte unregelmässige Herzaction; ferner die subjectiven Klagen der Kranken über das starke Herzlklopfen, die Kopfschmerzen, zunehmende Mattigkeit und verdriessliche Stimmung, die profusen Schweisse. Ausserdem halte ich für diagnostisch wichtig auch die Reihenfolge, in welcher die Symptome aufgetreten sind: zuerst Kopfschmerzen, dann Herzklopfen, hierauf die Entwickelung des Kropfes, später Mattigkeit, profuse Schweissbildung, und endlich die Beschwerden über das rechte Auge. Demnach ist eine durch Struma bedingte Compressionsneurose des Sympathicus im Sinne Eulenburg's (Berliner klinische Wochenschrift 1869) ausgeschlossen.

Die Frage, ob die erwähnten Krankheitserscheinungen mit der bei der ersten Untersuchung festgestellten Nasenaffection in $\mathrm{Zu}$ sammenhang standen, muss ich bejahen unter dem Druck der Thatsache, dass die beiden lästigsten, seit etwa vier Jahren bestehenden Symptome, der Kopfschmerz gleichzeitig mit der Operation, das Herzklopfen fünf Tage nach derselben vollständig beseitigt war. Ein Einfluss des in den ersten Tagen genommenen schwachen Eisenpräparates: Tr. Ferri pomat., Tr. Rhei vinos. ana, dreimal täglich $1 / 2$ Theelöffel, muss in so kurzer Zeit vollkommen ausgeschlossen werden. Ebenso wenig bin ich geneigt, auch der elektrischen Behandlung des Kropfes eine wesentliche Bedeutung für die Rückbildung desselben zuzuschreiben; denn erstens versicherte die Kranke nach zu kurzer Zeit, schon in der fünften Sitzung, dass sie eine Verkleinerung der Geschwulst bemerke, und zweitens hat die auffallende Abnahme der Struma gerade in der Zeit stattgefunden, wo die Kranke nicht elektrisirt worden ist. Gleichwohl glaube ich, dass zur Hebung des Allgemeinbefindens bis zu dem gegenwärtigen erfreulichen Zustande der Kranken die dauernde Einnahme von Eisen und schliesslich auch die Beseitigung des Gebärmutterleidens gewiss nicht unwesentlich beigetragen hat.

Bei der geringen Anzahl von derartigen Beobachtungen, welche 
nach dem Ausspruch eines so erfahrenen und gewichtigen Autors, wie B. Fraenkel, als durchaus exceptionelle Vorkommnisse zu betrachten sind, dürfte es sehr gewagt erscheinen, schon jetzt weitergehende Schlüsse zu ziehen. Aber auch die wenigen und seltenen Fälle zwingen uns zu der Annahme, dass auf der Basis einer von Eulenburg der Basedow'schen Krankheit unterstellten Asthenie auch periphere, von der Nase ausgehende, dauernde Erregungen imstande sind, gelegentlich das vielgedeutete Krankheitsbild des Morbus Basedowii zu erzeugen. Hack hat sich darüber im Anschluss an seinen Fall bereits bestimmt ausgesprochen und führt als Analoga für die oben erwähnten Fälle die vasodilatatorischen Reflexneurosen an: Asthma, Migräne, hartnäckige Conjunctivalhyperaemieen, Röthung der äusseren Nase, welche durch Zerstorrung hypertrophirter Schwellgewebe definitiv geheilt sind. In der That, bei dem eingehenden Studium der bisher beobachteten Nasenreflexneurosen, unter anderen von Hack, Scheinmann, und namentlich der zahlreichen sorgfältigen Beobachtungen von Sommerbrodt, kann man sich der Ansicht nicht verschliessen, dass der Unterschied zwischen hier und dort nur ein gradueller ist. 